\title{
Split-night versus full-night studies for sleep apnoea/hypopnoea syndrome
}

\author{
N. McArdle*, A. Grove ${ }^{\#}$ G. Devereux*, L. Mackay-Brown\# ${ }^{\#}$ T. Mackay*, N.J. Douglas*
}

\begin{abstract}
Split-night versus full-night studies for sleep apnoea/hypopnoea syndrome. N. McArdle, A. Grove, G. Devereux, L. Mackay-Brown, T. Mackay, N.J Douglas. (C)ERS Journals Ltd 2000 .

ABSTRACT: Investigation and treatment of sleep apnoea/hypopnoea syndrome (SAHS) is placing increasing demands on healthcare resources. This workload may be reduced by using split-night studies instead of the standard full-nights of diagnostic polysomnography and continuous positive airway pressure (CPAP) titration. Splitnight studies involve polysomnography in the first half of the night followed, if there is an abnormal frequency of apnoeas and hypopneas, by CPAP titration for the remainder of the night.

The authors' database of all patients prescribed a CPAP trial 1991-1997 was used to compare long-term outcomes in all 49 (46 accepting CPAP) patients prescribed split-night studies with those in full-night patients, matched 1:2 using an apnoea/ hypopnoea index (AHI) of $\pm 15 \%$ and Epworth score of \pm 3 units.

Classical symptoms of SAHS were the main reason for the split-night studies $(n=27)$. There were no differences between the groups in long-term CPAP use, median nightly CPAP use (split-night $6.0 \mathrm{~h} \cdot$ night $^{-1}$, interquartile range (IQR) 3.8-7.4, fullnight; 6.2 h.night ${ }^{-1}$, IQR 3.7-7.0, p=0.9), post-treatment Epworth scores and frequency of nursing interventions/clinic visits required. The median time from referral to treatment was less for the split-night patients (13 months, IQR 11-20 months) than for full-night patients (22 months, IQR 12-34 months; $p=0.003$ ).

Split-night studies, in selected patients, result in equivalent long-term continuous positive airway pressure use to full-night studies with shorter treatment times and less healthcare utilization.

Eur Respir J 2000; 15: 670-675.
\end{abstract}

Sleep apnoea/hypopnoea syndrome (SAHS) is a common condition, affecting $2-4 \%$ of the middle-aged population [1]. The consequences of SAHS include daytime sleepiness, altered mood and impaired cognitive function $[2,3]$ leading to reduced quality of life $[4,5]$ and an increased risk of accidents at work and on the road [6-8]. Many sleep centres are struggling to cope with the number of patients being referred and the financial restrictions placed on their investigation. One way of reducing the time taken to investigate and treat patients is to use splitnight studies whereby diagnostic polysomnography and continuous positive airway pressure (CPAP) titration are accomplished on the same night rather than the standard two nights consisting of a diagnostic polysomnography night and subsequent CPAP titration night (full-night patients)

Despite the potential savings, split-night studies are not widely used in all countries, at least partly because of concerns about the unknown long-term outcome of these studies [9]. The few evaluations that have included clinically relevant outcomes have not used a control group [10], or, when a control group was used, the numbers were small ( $\mathrm{n}=12$, in each group) and the outcome evaluation was limited to mean nightly CPAP use at the initial clinic visit [11]. Therefore, a larger scale study of the value of split-night studies was performed.
*Respiratory Medicine Unit and ${ }^{\#}$ Medical School, University of Edinburgh, UK.

Correspondence: N.J. Douglas

Respiratory Medicine Unit

Dept of Medicine

University of Edinburgh

Royal Infirmary of Edinburgh

Edinburgh EH3 9YW

UK

Fax: 441315363255

Keywords: Continuous positive airway pressure compliance

diagnosis of sleep apnoea/hypopnoea

syndrome

healthcare utilization

split-night studies

Received: June 231999

Accepted after revision January 192000
Methods

Using the authors' database of 1,211 patients booked for CPAP titration between 1986 and 1997 [12], a matched controlled comparison was undertaken using prospectively gathered data on CPAP use and patient outcomes. Every patient who had undergone a split-night study was matched with two patients who had undergone full-night diagnostic polysomnography followed by a full-night CPAP titration study. The matching of split-night studies with full-night studies was performed using the number of apnoeas and hypopneas per hour of sleep (apnoea/ hypopnoea index (AHI)) and the Epworth score, blind to the study outcomes. These variables were chosen as they had been found to be independent predictors of long-term CPAP use in studies involving this group of patients [12]. The analyses included all patients booked for a split-night study during the study period, providing that they had been given a pretreatment Epworth score (the Epworth score was not in use prior to 1991 [13]) and their diagnosis had been based on polysomnography.

All full-night patients were matched to split-night patients using the following two criteria: 1) AHI within 15\%, and 2) Epworth score within 3 units.

Patients were evaluated for the symptoms and signs of SAHS [14] by a physician experienced in sleep medicine. 
Split-night studies were performed after clinical evaluation in the presence of the following criteria: 1) "Classical" clinical features of SAHS; this required the presence of loud snoring, witnessed apnoeas and an unequivocal history of excessive daytime somnolence (including an Epworth score of >10). 2) A good history of SAHS and a supportive investigation from another centre. A supportive investigation included: suggestive overnight oximetry or a limited channel study (without electroencephalographic (EEG) recording) with an apnoea/hypopnoea rate $\cdot \mathrm{h}^{-1}$ recorded $(\mathrm{AH})$ of $>5$ but $<30$. In the authors' unit, patients with an $\mathrm{AH}$ of $>5$ but $<30$ in a limited study also require an EEG study for the diagnosis of SAHS. 3) A good history of SAHS plus logistical reasons (e.g. a long distance to travel for investigation).

Patients did not undergo split-night studies if there were symptoms suggestive of other sleep disorders, such as narcolepsy. During the diagnostic portion of the split-night study, the AHI was estimated by the specialist night nurse, after 2-3 h of sleep. If the diagnostic part of the study was thought by the nurse to show an AHI of $>20$, manual CPAP titration was undertaken. Formal scoring of the AHI in the split-night patients was not performed during the night, but was left until the following day. Full-night patients underwent a clinical evaluation and polysomnography prior to CPAP titration. They were booked for CPAP titration because they had two or more major symptoms of SAHS [14] and had an AHI of $>5$.

Polysomnography was performed using the authors' usual equipment and scoring techniques [15]. Apnoeas were defined as the absence of oronasal airflow in the presence of thoracoabdominal movement for $\geq 10 \mathrm{~s}$ and hypopnoeas as a $\geq 50 \%$ reduction in thoracoabdominal movement for $\geq 10 \mathrm{~s}$. Split-night patients were further classified according to whether or not the oxygen saturation trace, during the diagnostic portion of the assessment, was considered to be definitive for SAHS, i.e., showed repetitive dipping of oxygen levels on visual inspection [16]. Manual CPAP titration was performed to obtain the minimum pressure that normalized the breathing pattern and minimized EEG arousals [17].

All patients received an educational intervention prior to a CPAP titration night or a split-night study. The educational intervention involved an explanation of SAHS and CPAP treatment by medical staff at the clinic and by specialized CPAP nurses, an educational video and maskfitting from a wide range of mask types as well as $20 \mathrm{~min}$ spent acclimatizing to CPAP on a bed during the day. For patients booked for a split-night study, it was explained that CPAP treatment was likely to be needed, and, if so, would be initiated during the night. When CPAP was initiated during a split-night study, the CPAP mask was placed over the existing monitoring leads in order to minimize patient disturbance.

At follow-up, the mean nightly run time for each patient's CPAP machine was calculated from the time clock reading. Details of nursing interventions were obtained from the specialist CPAP nurses' records and clinical records. They were classified into eight categories: mask changes, pressure changes, topical nasal steroids, nasal ipratropium, other topical nasal agents, provision of humidifiers, telephone advice and "other" interventions. The total of all nursing interventions for each patient was also calculated. The median frequency of nursing interventions in each group was calculated using the number of nursing interventions as the numerator and the follow-up time as the denominator for each subject. A similar calculation was made for the frequency of clinic visits. The median time from referral to CPAP treatment was also calculated for each group.

\section{Statistical analysis}

Statistical analysis was performed using the SPSS statistical software package (SPSS Inc., Chicago, IL, USA). Nonparametric methods were used (Mann-Whitney U-test, Chi-squared test and Spearman rank correlation) in the analyses as the variables measured were dichotomous, categorical or not normally distributed. In order to analyse the effects of independent variables on long-term CPAP use, methods of survival (Kaplan-Meier) analysis were used [18]. Kaplan-Meier analysis allows for variable follow-up times, and, by using "censored" data, adjusts for events (such as death) that prevent the event of interest being reached (i.e. deliberate stopping of CPAP treatment). The log-rank test was used to compare the effect of dichotomous variables (e.g. study type: split-night versus full-night studies) on long-term CPAP use. To further evaluate the role of possible explanatory variables in long-term CPAP use in the split-night group, Cox's proportional hazards model $[18,19]$ was used. The use of Cox's method for survival analysis is analogous to the use of multiple regression as an extension of linear regression. For all analyses, statistical significance required a two-tailed $\mathrm{p}$-value of $<0.05$.

\section{Results}

Between 1991 and 1997, a total of 49 patients were booked for a split-night study. Of these, one patient failed to attend, one refused to take the CPAP device home after the study and one did not require CPAP treatment after the split-night study was converted to a full diagnostic night. The remaining 46 split-night patients were matched with 92 full-night patients, making a total of 138 subjects evaluated for long-term outcomes.

Patients underwent a split-night study because of a classical history of SAHS ( $n=27)$, a good history of SAHS and supportive limited investigation $(\mathrm{n}=13)$ or for logistical reasons $(n=6)$ (table 1$)$. Comparison of split-night patients with control patients showed they were well matched, with no difference at baseline in AHI, Epworth score, body mass index, age, sex, proportion of patients with classical symptoms of SAHS and CPAP titration pressure (table 2).

Table 1. - Reason for split-night study

\begin{tabular}{lccc}
\hline & \multicolumn{2}{c}{ Patients } & AHI* \\
\cline { 2 - 3 } & $\mathrm{n}$ & $\%$ & \\
\hline Classical history & 27 & 59 & $80(20-126)$ \\
$\begin{array}{l}\text { Good history and supportive } \\
\text { investigation }\end{array}$ & 13 & 28 & $40(22-64)$ \\
Logistical & 6 & 13 & $17(11-48)$ \\
\hline
\end{tabular}

*: median (interquartile range). AHI: apnoea/hypopnoea index. 
Table 2. - Baseline comparison of split-night and fullnight patients

\begin{tabular}{lccc}
\hline & Split-night & Full-night & p-value \\
\hline Male \% & 80 & 83 & 0.9 \\
Age yrs & $50(42-56)$ & $49(43-56)$ & 0.6 \\
BMI kg.m ${ }^{-2}$ & $34(27-42)$ & $32(28-39)$ & 0.4 \\
Epworth score & $15(10-19)$ & $15(9-18)$ & 0.7 \\
AHI & $49(18-98)$ & $49(18-103)$ & 0.9 \\
Classical SAHS & $27 / 46$ & $52 / 92$ & 0.8 \\
$\quad$ symptoms & & & \\
CPAP cmH $_{2} \mathrm{O}$ & $8.5(7.6-12)$ & $9.0(7.5-12)$ & 0.9 \\
\hline
\end{tabular}

Data are presented as median (interquartile range). BMI: body mass index; AHI: apnoea/hypopnoea index; SAHS: sleep apnoea/hypopnoea syndrome; CPAP: continuous positive airway pressure.

In the diagnostic phase, the total sleep time (TST), total recording time (TRT), and stage 1, stage 2, stage 3 and 4 rapid eye movement (REM) time were all shorter for the split-night group compared to the full night group (table 3 ). However, the sleep efficiency index (SEI), was the same in both groups (table 3). The TRT during CPAP titration was longer in the full-night group but the titration AHI (at the final titration pressure) was similar in the two groups (table 3).

Split-night patients were followed up for a median time of 22 months and full-night patients for a median of 27 months. During this time 10 of $46(22 \%)$ patients in the split-night group stopped CPAP treatment and a similar proportion of the full-night group (19 of 92 (21\%) patients) stopped. One patient in the full-night group died. More than one reason was given for stopping CPAP by some patients. Patients who underwent split-night studies stopped CPAP because of discomfort in six cases or because of lack of benefit in four. In the full-night group, patients stopped because of lack of benefit in 12 cases, discomfort in eight and/or other reasons in one. There was no difference between the groups in continued CPAP use (fig. 1, log-rank test $\mathrm{p}=0.6$ ), or in nightly CPAP usage among those continuing to use CPAP (table 4).

There were no differences between the split-night and full-night groups in the frequency of nursing interventions or clinic visits needed (table 4). Analysis of each of the

Table 3. - Comparison of polysomnographic results for split-night and full-night patients

\begin{tabular}{lccc}
\hline $\begin{array}{l}\text { Polysomnographic } \\
\text { data }\end{array}$ & Split-night & Full-night & p-value \\
\hline D TRT min & $131(116-170)$ & $462(422-481)$ & $<0.0001$ \\
D TST min & $104(68-134)$ & $322(229-386)$ & $<0.0001$ \\
Dst 1 min & $6(2-12)$ & $24(10-52)$ & $<0.0001$ \\
Dst 2 min & $66(41-86)$ & $170(120-235)$ & $<0.0001$ \\
Dst 3 \& 4 min & $20(0-33)$ & $34(8-66)$ & 0.0005 \\
Dst REM min & $13(0-21)$ & $49(31-73)$ & $<0.0001$ \\
D SEI \% & $74(57-83)$ & $74(58-83)$ & 0.8 \\
CPAP TRT min & $340(300-354)$ & $466(438-485)$ & $<0.0001$ \\
Titration AHI & $9(4-14)$ & $5(2-13)$ & 0.1 \\
\hline
\end{tabular}

Data are presented as median (interquartile range). D: diagnostic; Dst: diagnostic stage; TRT: total recording time; TST: total sleep time; REM: rapid eye movement; SEI: Sleep Efficiency Index; CPAP: continuous positive airway pressure; Titration AHI: titration apnoea/hypopnoea index.

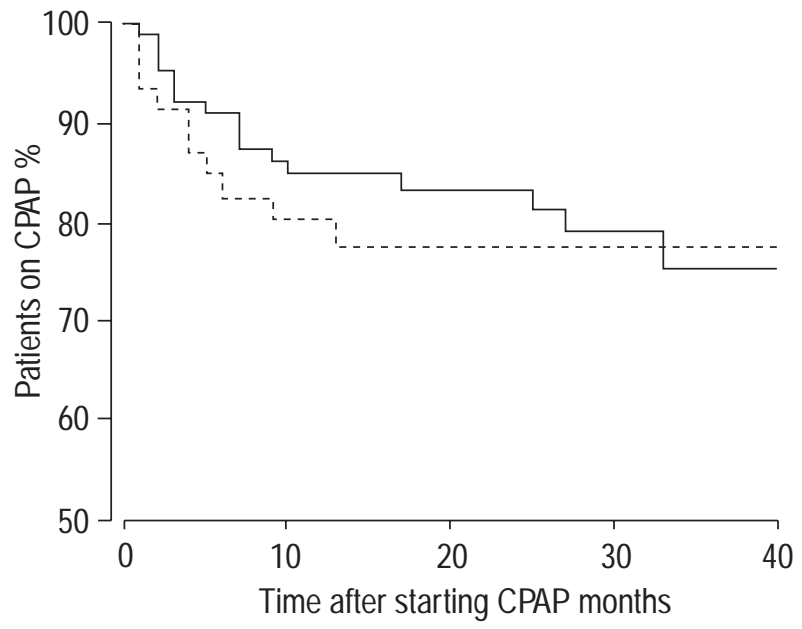

Fig. 1. - Long-term continuous positive airway pressure (CPAP) use: split-night $(---, n=46)$ versus full-night $(-, n=92)$.

eight categories of nursing interventions did not reveal any differences between the groups in any category of intervention. The post-treatment Epworth scores on patients who continued to use CPAP treatment, were similar in each group (table 4). The median time from referral to starting on CPAP was less for the split-night patients (13 months, interquartile range (IQR) 10-20) than for the full-night patients (22 months, IQR 11-34 months, $\mathrm{p}=$ 0.003).

Continuous positive airway pressure use in subgroups of patients

Five of the 46 patients in the split-night group required a further night in the sleep centre for CPAP titration. Two had their split-night study converted to a full diagnostic study and subsequently underwent a full CPAP titration night for mild severity disease (AHI of 12 and 13). Three required a second night of CPAP titration, because they were unable to tolerate split-night CPAP titration, and the same proportion of full-night patients required a second CPAP titration night (6 of 92). Analysis of the above outcome measures, using only the 41 split-night patients that did not require a further CPAP titration night (and their matched controls) did not alter any of the findings.

The effect of titration with and without a REM period at the final pressure on long-term CPAP use was evaluated in

Table 4. - Comparison of outcome measures for splitnight and full-night patients

\begin{tabular}{|c|c|c|c|}
\hline Outcome measures & Split-night & Full-night & p-value \\
\hline Nightly CPAP use $h \cdot$ night $^{-1}$ & $\begin{array}{c}6.0 \\
(3.8-7.4)\end{array}$ & $\begin{array}{c}6.2 \\
(3.7-7.0)\end{array}$ & 0.9 \\
\hline $\begin{array}{l}\text { Frequency nursing interven- } \\
\text { tions interventions } \cdot \mathrm{yr}^{-1}\end{array}$ & $\begin{array}{c}1.0 \\
(0-2.6)\end{array}$ & $\begin{array}{c}0.8 \\
(0.3-2.4)\end{array}$ & 0.9 \\
\hline $\begin{array}{l}\text { Frequency clinic visits } \\
\text { visits } \cdot \mathrm{yr}^{-1}\end{array}$ & $\begin{array}{c}2.3 \\
(1.7-2.9)\end{array}$ & $\begin{array}{c}2.0 \\
(1.4-2.7)\end{array}$ & 0.1 \\
\hline Epworth score on CPAP & $\begin{array}{c}7 \\
(3-12)\end{array}$ & $\begin{array}{c}9 \\
(4-13)\end{array}$ & 0.3 \\
\hline
\end{tabular}

Data are presented as median (interquartile range). CPAP: continuous positive airway pressure. 
the 41 patients whose CPAP was determined from the splitnight titration. Patients who had REM, at final pressure, during the titration study $(n=33)$ were similar to those that did not have REM ( $n=8)$ in their median AHI (REM: 64, IQR 19-111; No REM: 31, IQR 22-123; $p=0.9$ ) and median Epworth score (REM: 15, IQR 10-17.5; No REM: 17, IQR 6-20; $p=0.7)$. There were no differences in the Kaplan-Meier plots of continued CPAP use whether there was a REM period during the titration or not (log-rank test, $\mathrm{p}=0.3$ ). Additionally, there were no differences in median nightly CPAP use between these groups (REM: $6.5 \mathrm{~h}$. night $^{-1}$, IQR 4.2-7.7; No REM: $5.3 \mathrm{~h} \cdot$ night $^{-1}$, IQR 2.8-6.7, $\mathrm{p}=0.4)$.

Continued CPAP use in the subgroup with mild severity SAHS, which the authors define as an AHI of $\leq 30$, was similar in split-night $(\mathrm{n}=17)$ and full-night $(\mathrm{n}=34)$ patients (log-rank test $\mathrm{p}=0.5$ ) (fig. 2). The median nightly CPAP use in the mild SAHS patients who continued using CPAP was similar (split-night: $6.7 \mathrm{~h} \cdot$ night $^{-1}$, IQR 3.9-7.2 full-night: $6.2 \mathrm{~h} \cdot$ night $^{-1}$, IQR $4.6-6.8 ; \mathrm{p}=0.5$ ).

Cox's proportional hazards model showed that the independent determinants of continued CPAP use in the split-night patients were increasing pretreatment Epworth score and decreasing TST during the diagnostic half of the study. Patients with a typical pattern of oxygen desaturation $(n=21)$ had a shorter diagnostic TST (median $82 \mathrm{~min}$, IQR 57-112) than those $(n=20)$ without a typical pattern of desaturation (median 113 min, IQR 101-140) $(\mathrm{p}=0.002)$.

\section{Discussion}

The present study showed that in selected patients, most with classical symptoms of SAHS, a split-night study resulted in similar CPAP use and post-treatment Epworth score to standard full nights of diagnostic polysomnography and CPAP titration. The frequency of follow-up nursing interventions and clinic visits was the same for both groups, but, importantly, split-night patients had a shorter waiting time from referral to CPAP treatment. Patients with mild severity disease $(\mathrm{AHI} \leq 30)$ had similar CPAP use in the split-night and full-night groups. The absence of REM during CPAP pressure titration (at final pressure) was not

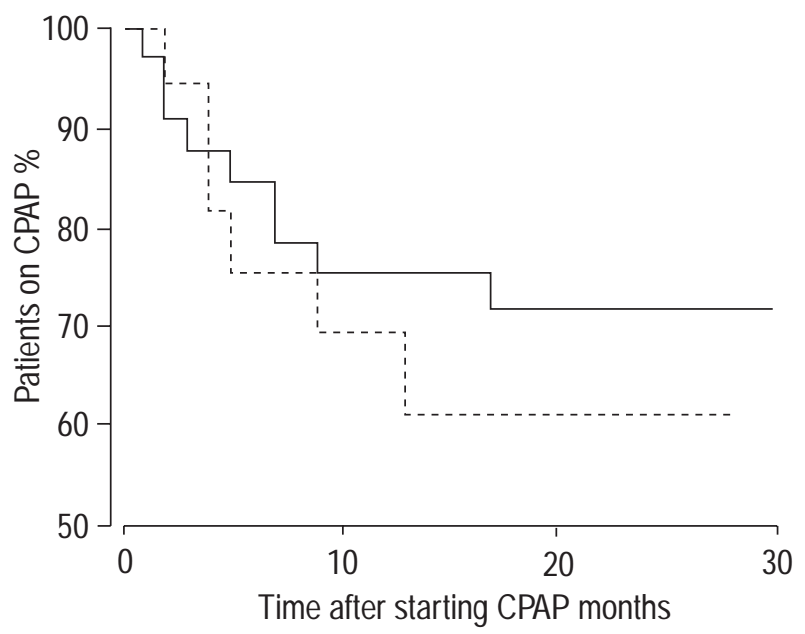

Fig. 2. - Long-term continuous positive airway pressure (CPAP) use in mild disease, with an apnoea/hypopnoea index of $\leq 30$. — : full-night, $n=34) ;-\cdots$ - : split-night $(\mathrm{n}=17)$. associated with lower CPAP use. The reduction in sleep centre overnight admissions without any increase in follow-up intervention suggests that the split-night protocol may result in significant cost savings.

There have been few reported assessments of outcomes of split-night studies. FLEURY et al. [10] evaluated 31 patients booked for split-night studies, with a mean CPAP nightly use of $6.7 \mathrm{~h}$ in those continuing to use CPAP $(\mathrm{n}=17)$. It was concluded that patients undergoing splitnight studies had similar CPAP use to those undergoing full-nights studies; however, this conclusion was based on uncontrolled historical comparisons with full-night studies. The split-night technique was believed to be reliable and cost saving, but no other assessment of effectiveness was made and there were no measurements of interventions needed. FLeURY et al. [10] found CPAP use among those taking the CPAP device home was $80 \%$, after a mean follow-up time of 9.5 months. The current study also found $80 \%$ of patients using CPAP at 9.5 months and found, after a longer follow-up of 22 months, that 78\% were still using CPAP. STROLLO et al. [11] performed a retrospective matched (for age, sex and severity of obstructive breathing) controlled comparison of split-night and full-night patients. In those patients accepting CPAP, the mean nightly duration of use, 4-6 weeks after start of CPAP was the only outcome measured. No differences were found between the groups in CPAP use; however, the number of patients studied was small (12 in each group) and a type 2 error cannot be excluded. Some of the shortcomings of the above studies have been addressed, in the present study by examining a larger group of patients with follow-up times of $\sim 2$ yrs and have included other relevant assessments such as follow-up Epworth score and the frequency of nursing and medical interventions needed.

In the current study, the split-night CPAP refusal rate (2 of $49,4 \%$ ) was similar to the reported refusal rate for all patients booked for CPAP between 1986 and 1997 (4.5\%) [12]. The refusal percentage of the current study of $4 \%$, however, was considerably lower than those previously reported for split-night studies $(22-37.5 \%)[10,11]$. The reason for this greater acceptance rate is uncertain, but may relate to the use of extensive education before all split-night studies, similar to that used before CPAP titration nights for full-night patients. In contrast, FLEURY et al. [10] did not report the use of education prior to splitnight studies and STROLlo et al. [11] reported only that patients were allowed to try a variety of commercially available masks prior to the study.

The proportion of patients requiring an additional CPAP titration night was similar in the split-night and full-night groups. There has been concern that the shorter time spent interacting with sleep centre staff in split-night studies adversely influences CPAP use [9]. Therefore, a separate analysis of outcomes was performed, excluding splitnight patients that had an extra CPAP titration night. This did not change any of the findings. Additionally, two patients in the split-study group underwent a suboptimal CPAP titration, with titration AHI of 60 and 70 . However, these patients had severe disease (AHI 195 and 180, Epworth score 17 and 13, respectively) and were keen to take the CPAP device home. They were managed without a further CPAP titration night and their follow-up Epworth scores were 5 and 9, respectively. Combining these 
patients with those that required a further full night of CPAP titration gave an "unsuccessful" split-night titration rate of 7 of $46(15 \%)$, similar to previously reported figures of 13 [10] and 15\% [11].

Stage REM is associated with more severe disturbances of upper airway function [20], and it has been suggested that adequate split-night studies should include REM sleep during CPAP titration at the final CPAP pressure [11]. In the split-night patients who did not need a second CPAP titration night, those with REM at the final titration pressure $(n=33)$ were compared with those without REM $(n=8)$. It was found that these two groups were of similar severity in baseline AHI and Epworth score and also showed similar CPAP use. These findings suggest that the presence of REM sleep may not be necessary for adequate CPAP pressure titration, although the authors accept that the number of patients studied without REM sleep is relatively small.

In keeping with American Thoracic Society recommendations [21], other investigators required an AHI of $>30$ in the diagnostic half of the split-night before proceeding to CPAP titration $[10,11]$. In the current study, 17 patients in the split-night group and 34 in the full-night group had an AHI of $\leq 30$ in the diagnostic study. There were no differences between these groups in median nightly CPAP use. Comparison of Kaplan-Meier plots suggests a trend towards worse long-term compliance in those patients who had undergone a split-night study (fig. 2), although this was not statistically significant. Larger patient numbers may be required to determine whether or not CPAP titration should proceed in the second half of the night when mild severity disease is evident during the diagnostic portion of the split-night study.

The independent predictors of long-term CPAP use were increasing pretreatment Epworth score and decreasing TST in the diagnostic portion of the study. Pretreatment Epworth score and other measures of subjective daytime sleepiness are established predictors of CPAP compliance $[12,22,23]$. The reason that a short diagnostic TST leads to better long-term use may be because this allows a longer time for CPAP titration, which may lead to a more accurate CPAP determination [24]. However, a typical pattern of oxygen desaturation is associated with a shorter diagnostic TST, presumably because this makes it easier for the night nurses to decide to start CPAP titration. A shorter diagnostic TST may lead to better long-term use because oxygen desaturation is a marker of disease severity and a predictor of CPAP compliance [22, 25].

Patients were not booked for split-night studies if there were symptoms of restless leg syndrome or suggestive of a diagnosis of narcolepsy. However, five patients had an elevated AHI and an increased frequency of leg jerks $(>5$ jerks $\cdot \mathrm{h}^{-1}$ ) during the diagnostic half of the split-night study (as did seven patients in the full-night group). These patients were treated with CPAP alone, with resolution of daytime sleepiness (post-treatment Epworth score of $\leq 7$ ). Four patients had symptoms that could be due to narcolepsy or SAHS [26], either vivid dreams or definite hypnagogic hallucinations. All these patients had classical symptoms of SAHS and markedly raised AHIs in the diagnostic portion of the split-night study (AHI 50-195) and showed reversal of daytime sleepiness with CPAP alone (post-treatment Epworth score of $\leq 5$ ). Further diag- nostic studies for narcolepsy have not been indicated in these patients.

The reasons for patients undergoing split-night studies were divided into three categories, with most studies being carried out because of a "classical" history of SAHS. The present findings are most applicable to patients who present with classical symptoms of SAHS, as defined above. The other main clinical group of patients studied had a good history of SAHS as well as having undergone a supportive limited investigation (oximetry or AutoSet (ResMed Ltd., Abingdon, UK)). Although the number in this group was smaller, the reason for the split-night study was not an independent predictor of CPAP use, indicating that these patients can be managed effectively with a split-night study.

The limitations of this study include the use of retrospective data. However, all data were accrued prospectively, reducing the bias from the retrospective analysis of studies. Furthermore, matching was performed using the split-night apnoea/hypopnoea index and Epworth scores, blinded to patient study outcomes. Because of the lack of good evidence for the effectiveness of split-night studies, only 49 patients were booked during the study period. Nevertheless, the possibility of a selection bias seems unlikely since the patients booked for split-night studies were similar to matched full-night study patients in important clinical characteristics (age, sex, Epworth score, symptom severity and body mass index) used to assess patients for the presence of sleep apnoea/hypopnoea syndrome (table 2). Prospective randomized controlled trials would be useful to confirm that the outcome of split-night studies, in selected patients, is equivalent to two full nights of assessment.

\section{References}

1. Young T, Palta M, Dempsey J, Skatrud J, Weber S, Badr S. The occurrence of sleep-disordered breathing among middle-aged adults. N Engl J Med 1993; 328: 1230-1235.

2. Cheshire K, Engleman H, Deary I, Shapiro C, Douglas N. Factors impairing daytime performance in patients with sleep apnea/hypopnea syndrome. Arch Intern Med 1992; 152: 538-541.

3. Guilleminault C, Van der Hoed J, Mitler M. Clinical overview of the sleep apnea syndromes. In: Guilleminault C, Dement WC, eds. Sleep Apnea Syndromes. New York, Alan R. Liss, 1978; pp. 1-12.

4. Smith I, Shneerson J. Is the SF36 sensitive to sleep disruption? A study in subjects with sleep apnoea. J Sleep Res 1995; 4: 183-188.

5. Jenkinson C, Stradling J, Peterson S. Comparison of three measures of quality of life outcome in the evaluation of continuous positive airways pressure therapy for sleep apnoea. J Sleep Res 1997; 6: 199-204.

6. Findley L, Unverzagt M, Suratt P. Automobile accidents involving patients with obstructive sleep apnea. Am Rev Respir Dis 1988; 138: 337-340.

7. George C, Nickerson P, Hanly P, Millar T, Kryger M. Sleep apnea patients have more automobile accidents. Lancet 1987; 2: 447.

8. Teran-Santos J, Jimenez-Gomez A, Cordero-Guevara J, and the Cooperative Group Burgos-Santander. The association between sleep apnea and the risk of traffic accidents. N Engl J Med 1999; 340: 847-851. 
9. Jamieson A. Split-night studies: a new standard? Forcing the examination of outcome. Sleep 1991; 14: 381-382.

10. Fleury B, Rakotonanharv D, Tehindrazanarivelo A, Hausser-Hauw C, Lebeau B. Long-term compliance to continuous positive airway pressure therapy (nCPAP) set up during a split-night polysomnography. Sleep 1994; 17: 512-515.

11. Strollo P, Sanders M, Constantino J, Walsh S, Stiller R, Atwood C. Split-night studies for the diagnosis and treatment of sleep-disordered breathing. Sleep 1996; 19: S255S259.

12. McArdle N, Devereux G, Heidarnejad H, Engleman H, Mackay T, Douglas N. Long-term use of CPAP therapy for sleep apnea/hypopnea syndrome. Am J Respir Crit Care Med 1999; 159: 1108-1114.

13. Johns MW. A new method for measuring daytime sleepiness: The Epworth sleepiness scale. Sleep 1991; 14: 540545.

14. Whyte K, Allen M, Jeffrey A, Gould G, Douglas N. Clinical features of the sleep apnoea/hypopnoea syndrome. $Q J$ Med 1989; 267: 659-666.

15. Gould G, Whyte K, Rhind G, et al. The sleep hypopnoea syndrome. Am Rev Respir Dis 1988; 137: 895-898.

16. Douglas N, Thomas S, Jan M. Clinical value of polysomnography. Lancet 1992; 339: 347-350.

17. Engleman HM, Martin SE, Douglas NJ. Compliance with CPAP therapy in patients with the sleep apnoea/hypnoea syndrome. Thorax 1994; 49: 263-266.
18. Altman D. Practical Statistics for Medical Research. London, Chapman and Hall, 1991; pp. 365-395.

19. Cox D. Regression models and life tables. J R Stat Soc B 1972; 34: 187-220.

20. Charbonneau M, Martin J, Olha A, Kimoff J, Levy R, Cosio M. Changes on obstructive sleep apnea characteristics through the night. Chest 1994; 106: 1695-1701.

21. Philipson E, Remmers J, and participants. Indications and standards for cardiopulmonary sleep studies. Am Rev Respir Dis 1989; 139: 559-568.

22. Rolfe I, Olson LG, Saunders NA. Long-term acceptance of continuous positive airway pressure in obstructive sleep apnea. Am J Respir Crit Care Med 1991; 144: 1130-1133.

23. Waldhorn R, Herrick T, Nguyen M, O'Donnell A, Sodero J, Potolicchio S. Long-term compliance with nasal continuous positive airway pressure therapy of obstructive sleep apnea. Chest 1990; 97: 33-38.

24. Yamashiro Y, Kryger M. CPAP titration for sleep apnea using a split-night protocol. Chest 1995; 107: 62-66.

25. Pieters T, Collard P, Aubert G, Dury M, Delguste P, Rodenstein DO. Acceptance and long-term compliance with nCPAP in patients with obstructive sleep apnoea syndrome. Eur Respir J 1996; 9: 939-944.

26. Guilleminault C. Clinical features and evaluation of obstructive sleep apnea. In: Kryger MH, Roth T, Dement W, eds. Principles and Practice of Sleep Medicine. Philadelphia, W.B. Saunders, 1989; p. 552-558. 